\title{
Library for First-Principle Models of Proton Exchange Membrane Fuel Cells in Modelica
}

\author{
Kevin L. Davies Christiaan J.J. Paredis Comas L. Haynes \\ Georgia Institute of Technology \\ Atlanta, Georgia USA
}

\begin{abstract}
This paper describes the architecture and key equations of FCSys, a library to model proton exchange membrane fuel cells (PEMFCs) in Modelica. The motivating goal of this work is to reconcile many of the published models of PEMFCs and combine them in a reconfigurable PEMFC model that is effective for a variety of uses. It is necessary to distill equations from fuel cell literature into forms that at once capture the essence of the physical interactions, are conducive to the physical modularity of the device, and work within the constraints and take full advantage of the Modelica language.
\end{abstract}

Since the behavior of PEMFCs depends on both advection and diffusion, a suitable alternative to the Modelica Fluid library and the stream concept is necessary. The proposed solution uses a "mixing" scheme based on the exponential of the Péclet numbers for each transport process. Storage and transport processes are co-located in each subregion of a rectilinear grid-all in the same base model. The Onsager formulation is used, whereby the effort and flow rate are conjugates of the entropy flow rate associated with energy transfer.

The implementation is modular. It allows species to be enabled independently for each region. In addition, the geometric axes may be independently enabled (up to 3D) and shearing (transverse momentum) may be optionally included. Chemical/electrochemical interactions are communicated in a fully acausal manner through expandable connectors.

This paper focuses on the motivation, background, and approach. Future publications will describe the ongoing work to calibrate, validate, and utilize the model for particular case studies. The library is made available as open source.

Keywords: PEMFC; three dimensional; fluid dynamics; electrochemistry; heat transfer; advection; diffusion; momentum; Onsager

\section{Introduction}

In certain power applications, fuel cell (FC) systems are preferable because they can convert fuel energy to work more efficiently than internal combustion engines and have energy-to-power ratios that can be easily adapted, unlike batteries. A FC system can be refueled quickly like an internal combustion engine (ICE) system, or it can be designed to recharge like a battery by operating in electrolysis mode [4]. Of the various fuel cell technologies, PEMFCs are best suited to meet the power-cycling and packaging requirements of vehicles and portable devices.

However, the cost and durability, and to a lesser extent, size and weight, of PEMFCs are not yet adequate to justify their use beyond niche devices and select demonstrations. Much work is being done to investigate the modes of failure and degradation, develop new materials and structures, improve manufacturing processes, and design better systems [26]. Mathematical models of PEMFCs are being used to help understand the relevant physical phenomena, study the effects of design choices, and perform model-based control. The breadth of these goals has led to a multitude of specialized models.

PEMFCs have a solid polymer-based electrolyte (the PEM) and operate at low temperatures (typically below $100^{\circ} \mathrm{C}$ ). As shown in Figure 1, a singlecell PEMFC has few core components: PEM, electrodes, gas diffusion layers (GDLs), and flow plates [14]. However, most applications require a higher electrochemical potential difference than a single-cell PEMFC can provide; therefore, two or more cells are joined back-to-back to form a PEMFC stack.

A PEMFC operates on the electrochemical energy released by the reaction of hydrogen and oxygen to produce water (Eq. 1c). Its PEM (electrolyte) controls the reaction by selectively passing protons while acting as a barrier layer to hydrogen, oxygen, and electrons (see Fig. 1). This forces the reaction to occur 


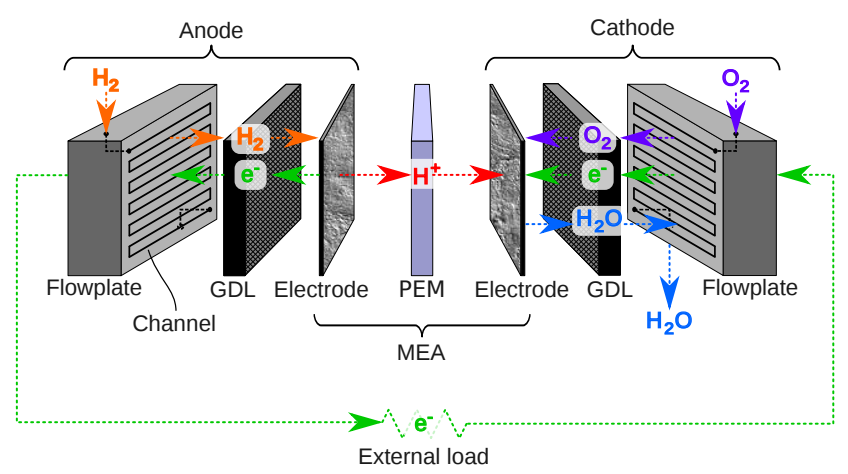

Figure 1: Layers of a single-cell PEMFC and the primary paths of hydrogen $\left(\mathrm{H}_{2}\right)$, oxygen $\left(\mathrm{O}_{2}\right)$, protons $\left(\mathrm{H}^{+}\right)$, electrons $\left(\mathrm{H}^{+}\right)$, and water $\left(\mathrm{H}_{2} \mathrm{O}\right)$ during normal operation

in two sub-reactions: the hydrogen oxidation reaction (HOR) whereby hydrogen is consumed and protons and electrons are produced (Eq. 1a) and the oxygen reduction reaction (ORR) whereby oxygen, protons, and electrons are consumed and water is produced (Eq. 1b). In order to complete the full reaction, the electrons must traverse an external path. The path is provided by an external load which can harness the energy of the net reaction.

$$
\begin{aligned}
2\left(\mathrm{H}_{2}\right. & \left.\rightarrow 2 \mathrm{H}^{+}+2 \mathrm{e}^{-}\right) \\
4 \mathrm{H}^{+}+4 \mathrm{e}^{-}+\mathrm{O}_{2} & \rightarrow 2 \mathrm{H}_{2} \mathrm{O} \\
\hline 2 \mathrm{H}_{2}+\mathrm{O}_{2} & \rightarrow 2 \mathrm{H}_{2} \mathrm{O}
\end{aligned}
$$

A broadly applicable PEMFC model library would need to contain models that are physically representative, meaning their predictions of behavior match reality (i.e., accuracy) and their structure corresponds to the physical domain. The PEMFC model library should approximate the dynamic voltage-current response of actual cells at nominal operating conditions and varying large signal electrical currents (e.g., [27, p. 3787 , Figs. $2 \mathrm{~b}$ and $2 \mathrm{c}]$ ). It should capture the operational effects of design parameters including component sizes and material properties (for hardware analysis and design) and should be capable of linearization (for control analysis and design). It should be able to describe relevant phenomena including electrochemical reactions, chemical/electrochemical transport, heat transport, and heat generation. It should have variable fidelity, that is, degree of spatial, dynamic, or behavioral detail. Finally, it should be modular, meaning its components can be interconnected in various ways to build models of larger systems. Unfortunately, however, no current PEMFC model library can provide these features and capabilities over the required range of operating conditions.

\section{Related work}

For reasons elaborated later, the acausal formalism and the Modelica language in particular is ideal for a dynamic, variable-fidelity, modular, and systemsoriented model of a PEMFC. There are hundreds of published PEMFC models [28], yet most of these use computational fluid dynamics (CFD) or causal (signalbased) models. Only four acausal, dynamic, and celllevel FC models are known to have been published; three are of PEMFCs and one is of a solid oxide fuel cell (SOFC).

Rubio et al. openly shared a 1D (through-the-cell) declarative PEMFC model which includes electroosmotic drag, double layer capacitance, variable choice of assumptions, and detailed diffusion with pore and species interactions (Knudsen flow and Maxwell-Stefan eqs.). However, the model is isothermal, does not include heat generation or a model of the flow plate, and only interacts with its surroundings electrically (no external thermal or fluid terminals) $[22,23]$.

Davies and Moore published a quasi-2D (throughthe-cell and along-the-channel) declarative PEMFC model which includes material and heat transport and storage, electro-osmotic drag, and variable choice of assumptions. However, the models of the cell's layers are not defined in a physical manner; for example, the electrode layers do not include chemical transport (only reactions and charge transport) [7, 8]. The last published version was based on the Modelica Fluid library [9]. As a result, it raised concerns (at the $7^{\text {th }}$ Modelica Conference) and had issues related to the integration of advection and diffusion, since Modelica Fluid offers a solution that is limited to purely advective flow.

McCain et al. implemented the model of McKay et al. [18] (mentioned previously) within a declarative formalism in order to linearize the model for control studies. However, the sub-models of the chemical species do not interact except in the flow plates and the PEM [17].

Salogni and Colonna published a 1D (along-thechannel) declarative model of a SOFC. It is wellconstructed, but since it treats each anode-to-cathode section of the cell as a integrated unit, its modularity does not resolve the physical layers of the cell [24].

A related approach is chemical bond graphs. Bond graphs have been used for decades to chemical reactions [5] and even applied to fuel cells [3, p. 355]. They are physical (in terms of energy) and are useful to trace causality, but they are not acausal. According 
to Cellier, bond graphs have not yet been successfully applied to problems in fluid dynamics. The reason is that fluid systems require mass conservation in addition to energy conservation [5, p. 331].

\section{Architecture}

The present model is described in differential algebraic equations (DAEs). Spatial variances are represented in terms of differences rather than derivatives. As stated by Mattiussi [15, pp. 2-3], this representation has three advantages: (i) it provides a unified perspective that is appropriate for many theories, (ii) it directly correlates the discretization of the physical region and the structural properties of the applied theories, and (iii) it is based on intuitive geometrical and physical concepts that help distinguish the numerical methods (e.g., finite difference method, finite volume method, and finite element method) from the underlying theories. In addition, powerful modeling tools (e.g., Dymola [12]) exist that can solve a model for the imposed causality, partition a dynamic model into the most numerically efficient systems of algebraic equations (i.e., resolve algebraic loops through tearing), perform index reduction (i.e., eliminate structural singularities), and linearize a model. Ultimately, this can result in a flexible and robust model that simulates quickly.

Table 1 summarizes the four base types of connectors that are used in the Modelica implementation. Figure 2 shows the hierarchy of the connectors, with the lowest level at the bottom. The flows of the material, linear momentum, and energy connectors are the rates of those quantities. The flow variable of the volume connector is the volume itself (not the volume flow rate). This allows the volume connector to be used to impose additivity of volume or Amagat's law - that the sum of the total volume of the region is the partial volumes of the species evaluated at the total pressure [20]. The effort variable is chosen such that the product of effort and the rate of the quantity is the entropy flow rate associated with the energy transfer. This approach is convenient for representing behavior in terms of Onsager reciprocal relations [1], as shown below. However, it departs from the traditional approach of power conjugate variables, which are generally used in the Package Modelica (exceptions include the rotational, translational, and thermal libraries) [19].

The physical quantities and units are represented using the approach described in [10]. Using that ap-

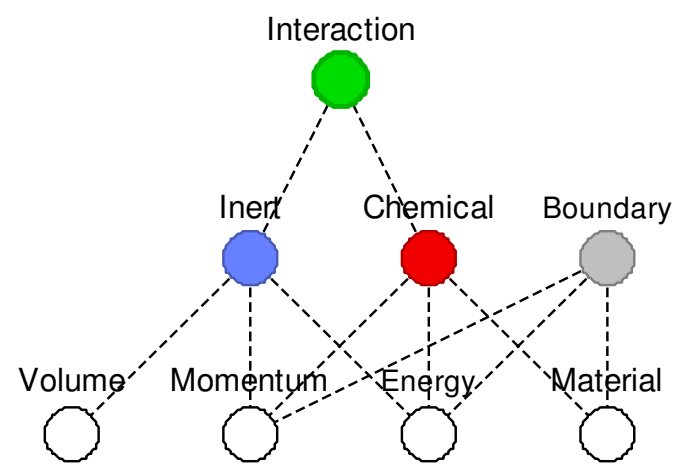

Figure 2: Hierarchy of the connectors

proach, the gas constant and the Faraday constant are both normalized to one. This simplifies the expression of the equations and allows electrons to be described in the same manner as other electrochemical species.

The model contains multiple rectilinear subregions of fixed length (and volume). Each subregion is an instance of the model shown in Figure 5b. Each of the region's six faces contains a bus connector (expandable). The bus may be populated with a sub-bus for each chemical or electrochemical species present in the region. Optionally, the sub-buses may be first grouped into buses for the mixtures or phases. By default, the length of the vector of momentum connectors is one-representing only normal velocity and force. However, the transverse directions may be included as well; the models adapt accordingly.

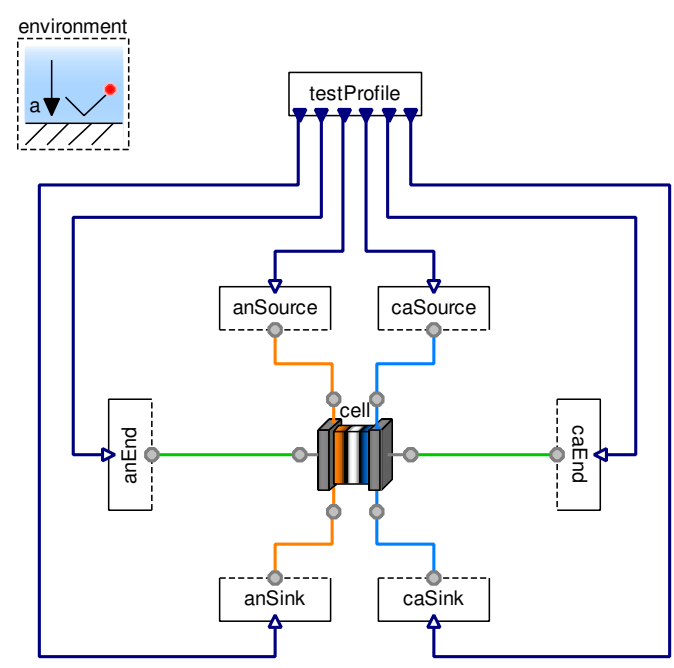

Figure 3: Diagram of a test model that imposes boundary conditions on the cell

In addition to the connectors, the subregion model may contain instances of models to represent species, reactions, and the total volume. A species model describes the advection, diffusion, and storage of material and momenta for a single electrochemical species $\left(\mathrm{e}^{-}, \mathrm{H}^{+}, \mathrm{H}_{2}, \mathrm{H}_{2} \mathrm{O}, \mathrm{N}_{2}\right.$, or $\left.\mathrm{O}_{2}\right)$. The species model con- 


\begin{tabular}{|c|c|c|c|}
\hline Within Icon(s) & Name/Quantity & Flow & Effort \\
\hline & Volume & $\begin{array}{l}\text { Volume } \\
V\left[\mathrm{~L}^{3}\right]\end{array}$ & $\begin{array}{l}\text { Pressure per temperature } \\
P / T\left[\mathrm{~N} \mathrm{~L}^{-3}\right]\end{array}$ \\
\hline & Linear momentum & $\begin{array}{l}\text { Force } \\
m \dot{\Phi}\left[\mathrm{ML} \mathrm{T}^{-2}\right]\end{array}$ & $\begin{array}{l}\text { Velocity per temperature } \\
\phi / T\left[\mathrm{NT} \mathrm{L} \mathrm{L}^{-1} \mathrm{M}^{-1}\right]\end{array}$ \\
\hline & Energy & $\begin{array}{l}\text { Power } \\
\dot{U}\left[\mathrm{~L}^{2} \mathrm{M} \mathrm{T}^{-3}\right]\end{array}$ & $\begin{array}{l}\text { Reciprocal of temperature } \\
1 / T\left[\mathrm{~N} \mathrm{~T}^{2} \mathrm{~L}^{-2} \mathrm{M}^{-1}\right]\end{array}$ \\
\hline & Material & $\begin{array}{l}\text { Current } \\
\dot{N}\left[\mathrm{~N} \mathrm{~T}^{-1}\right]\end{array}$ & $\begin{array}{l}\text { Chemical potential per temperature } \\
\mu / T[1]\end{array}$ \\
\hline
\end{tabular}

Table 1: Summary of connectors used in the models. The dimensions are noted in terms of mass (M), length $(\mathrm{L})$, time $(\mathrm{T})$, and particle number $(\mathrm{N})$. Since the gas constant and the Faraday constant are both normalized to one, charge and thermodynamic temperature are not taken to be independent dimensions.

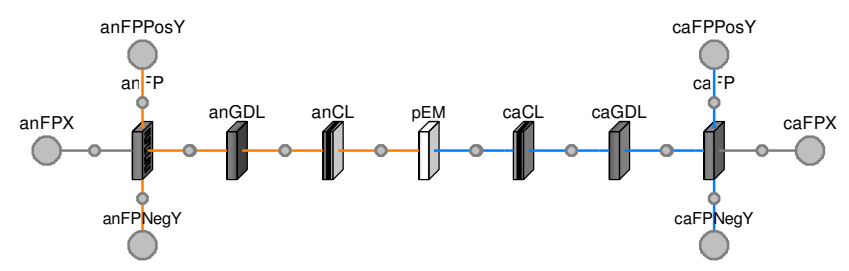

Figure 4: Diagram of a quasi-2D cell

nects to the boundaries and the interaction connector. Optionally, the species models may be nested within a mixture models, in which case the connections to the boundaries are indirect. The species, mixture, and subregion models allow the Cartesian axes to be enabled independently (by parameter), as long as one axis is enabled. As such, the boundary bus connectors of the subregion and mixture models are conditional. The array of boundary connectors in the species model has size $\{2,1\},\{2,2\}$, or $\{2,3\}$, where the first index represents the face ( 1 or 2$)$ and the second index represents one of the enabled axes.

The species are connected through the expandable interaction connector. In the connection, each species's chemical connector is named by the chemical formula of the species. The inert connector is simply named "inert." In order to prevent nonlinear systems of equations, the connection among species is mathematically direct. Each of the species interacts as if all other species were the same. For instance, each gaseous species interacts equally well with other gaseous species as with the solid. Stated alternatively, the species are "colorblind," which, in the case of volume, is consistent with the basis of Amagat's law [29]. While this is a strong assumption, it can be alleviated by choosing smaller regions, especially where the subregion boundaries are at or near the phase boundaries.

A reaction model exchanges material, momentum, and energy among multiple species. The reaction models may be used to model chemical or electrochemical reactions. In the chemical case, no material, momentum, or energy is stored. Then, the reaction model simply imposes the stoichiometric constraints (conservation of material), momentum rate balances (without loss), and energy rate balance. Chemical potentials, velocities, and temperatures, are equal in the chemical reaction model. There is no irreversibility; all of the loss is included in the instances of the species model. In the case of an electrochemical reaction, the electrochemical double-layer capacitance is included to account for the electrostatic potential. In the case of the HOR, electrons and protons are stored in equal amounts. Since there is no loss in the reactions models, the net reactions may be partitioned according to convenience, with no mathematical effect. For example, the ORR (Eq. 1b) is modeled as the net PEMFC reaction (Eq. 1c) and the HOR in reverse (Eq. 1a). Since $\mathrm{H}_{2}$ is not present in the cathode according to the model, it is only an intermediate step without storage and without loss. If additional species are present and interacting (e.g., $\mathrm{H}_{2} \mathrm{O}_{2}$ ), they must be included as instances of the species model and joined with the appropriate side-reactions.

At the top level of the subregion, an instance of the volume model is included. It connects to the "inert" sub-connector of the interaction bus to subtract the total volume of the region. Since volume is the flow variable, the partial volumes of the species must sum to the total volume.

Multiple instances of the subregion model are arranged and connected in up to three dimensions to create a region. Figure 5a shows a region, where the subRegions icon represents a 3D array of subregions. The layers of the PEMFC are regions. They are connected as shown in Figure 4 to create the cell model. 


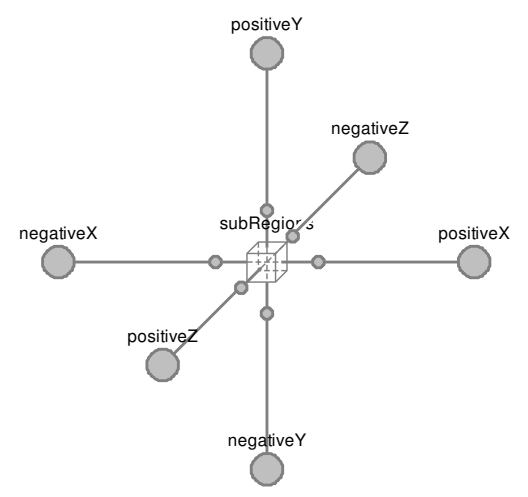

(a) Region or layer

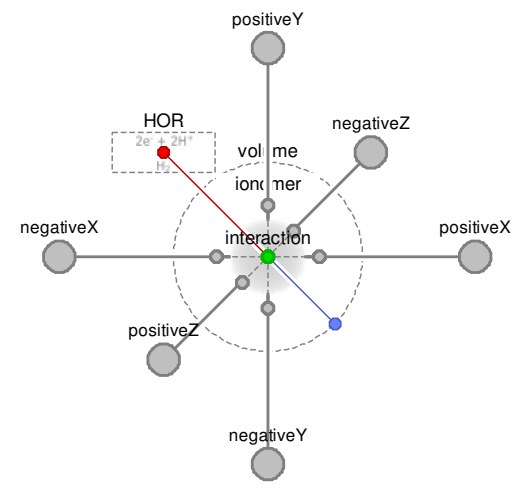

(b) Subregion

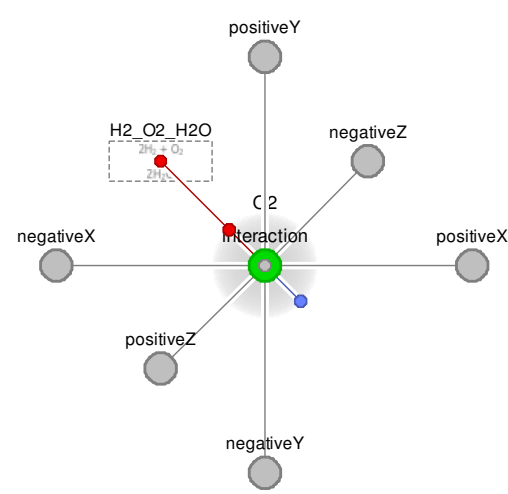

(c) Gaseous mixture. Others are mixtures are graphite and ionomer.

Figure 5: Diagrams of low-level models At the top/test level of the model, shown in Figure 3, an instance of the cell model is connected to models that impose boundary conditions.

\section{Equations}

\subsection{Physical characteristics}

The thermodynamic properties are implemented using the approach of McBride et al. [16], which gives specific heat capacities at constant pressure as seventhorder polynomials of temperature. These are the correlations which are used for ideal gases in the Modelica Media library. The pressure-volume-temperature cor- relations are implemented using the virial equation of state in the form that is explicit in specific volume [11]. That way, incompressible species and ideal gases can be represented by the same equation with only changes to the constants.

The generalized resistivities for material, momentum, energy, and volume are gathered from a multitude of sources. First, the rigid-sphere assumption may be used from kinetic theory [21]. Second, the correlations of NASA Glenn (formerly Lewis) are implemented where available for the momentum and thermal resistivity (from viscosity and thermal conductivity) $[25,25]$. Finally, property tables may used to set parameters (e.g., [13]). The implementation allows any of these options.

\subsection{Species Model}

Material is exchanged or transported into port $i$ according to equation $2 \mathrm{a}$, where $A_{j}, L_{j}$ and $\boldsymbol{\Phi}_{j}$ are the length and linear momentum along the axis of transport, respectively. The generalized material resistivity is $\Gamma_{N}$. The effective cross-sectional area is the product of the geometric cross-sectional area $\left(A_{j}\right)$ and a factor $(k)$ that accounts for roughness, porosity, tortuosity, and similar properties of the solid structure through which the transport occurs. The parameters $\beta$ are the Onsager coupling coefficients. By Onsager reciprocal theory, the coefficients $\beta_{i j}$ equals $\beta_{j i}$, where $i$ and $j$ are indexes to the quantities selected according to the theory [1]. The other variables in the equation are efforts and flows from Table 1. 


$$
\begin{aligned}
& \frac{L_{j}}{k A_{j}} \Gamma_{N}\left[\dot{N}_{i}+\beta_{N V}\left(\frac{P_{i}}{T_{i}}-\frac{P}{T}\right)-\boldsymbol{\beta}_{N \Phi}\left(\frac{\boldsymbol{\phi}_{i}}{T_{i}}-\frac{\boldsymbol{\phi}}{T}\right)+\beta_{N U}\left(\frac{1}{T_{i}}-\frac{1}{T}\right)\right]=\left(\frac{\mu_{i}}{T_{i}}-\frac{\mu}{T}\right)\left(1+e^{ \pm \boldsymbol{\Phi}_{j} \Gamma_{N} / k A_{j}}\right) \\
& \frac{L_{j}}{k A_{j}} \frac{\Gamma_{V} P}{v T}\left[\frac{\delta V}{\delta t}-\beta_{V N}\left(\frac{\mu_{i}}{T_{i}}-\frac{\mu}{T}\right)-\boldsymbol{\beta}_{V \boldsymbol{\Phi}}\left(\frac{\boldsymbol{\phi}_{i}}{T_{i}}+\frac{\boldsymbol{\phi}}{T}\right)+\beta_{V U}\left(\frac{1}{T_{i}}-\frac{1}{T}\right)\right]=-\left(\frac{P_{i}}{T_{i}}-\frac{P}{T}\right)\left(1+e^{ \pm \boldsymbol{\Phi}_{j} \Gamma_{V} / k A_{j}}\right) \\
& \frac{L_{j}}{k A_{j}} \frac{\boldsymbol{\Gamma}_{\boldsymbol{\Phi} j}}{m T}\left[m \dot{\boldsymbol{\Phi}}_{i}+\beta_{\boldsymbol{\Phi} V}\left(\frac{P_{i}}{T_{i}}-\frac{P}{T}\right)-\boldsymbol{\beta}_{\boldsymbol{\Phi} N}\left(\frac{\mu_{i}}{T_{i}}-\frac{\mu}{T}\right)+\beta_{\boldsymbol{\Phi} U}\left(\frac{1}{T_{i}}-\frac{1}{T}\right)\right]=\left(\frac{\boldsymbol{\phi}_{i}}{T_{i}}-\frac{\boldsymbol{\phi}}{T}\right)\left(1+e^{ \pm \boldsymbol{\Phi}_{j} \Gamma_{\boldsymbol{\Phi} j} / k A_{j}}\right) \\
& \frac{L_{j}}{k A_{j}} \frac{\Gamma_{U}}{T^{2}}\left[\dot{U}_{i}+\beta_{U V}\left(\frac{P_{i}}{T_{i}}-\frac{P}{T}\right)-\boldsymbol{\beta}_{U \boldsymbol{\Phi}}\left(\frac{\boldsymbol{\phi}_{i}}{T_{i}}-\frac{\boldsymbol{\phi}}{T}\right)-\beta_{U N}\left(\frac{\mu_{i}}{T_{i}}-\frac{\mu}{T}\right)\right]=-\left(\frac{1}{T_{i}}-\frac{1}{T}\right)\left(1+e^{ \pm \boldsymbol{\Phi}_{j} \Gamma_{U} / k A_{j}}\right) \\
& \frac{\delta N}{\delta t}=\sum \dot{N}_{i} \\
& \frac{\delta m \boldsymbol{\Phi}}{\delta t}+m N \mathbf{a}=\sum m \dot{\boldsymbol{\Phi}}_{i} \\
& \underbrace{c_{P} \frac{\delta T}{\delta t}}_{\text {thermal }}+\underbrace{P \frac{\delta V}{\delta t}}_{\text {volumetric }}-\underbrace{\boldsymbol{\phi} \frac{\delta m \boldsymbol{\Phi}}{\delta t}}_{\text {mechanical }}-\underbrace{\mu \frac{\delta N}{\delta t}}_{\text {electrochem. }}=T(\underbrace{\frac{1}{T_{i}} \dot{U}_{i}}_{\text {thermal }}+\underbrace{\frac{P_{i}}{T_{i}} \frac{\delta V}{\delta t}}_{\text {volumetric }}-\underbrace{\frac{\phi_{i}}{T_{i}} \dot{m}_{i}}_{\text {mechanical }}-\underbrace{\frac{\mu_{i}}{T_{i}} \dot{N}_{i}}_{\text {electrochem. }})
\end{aligned}
$$

The factors of the form $\left(1+\exp \left( \pm \boldsymbol{\Phi}_{j} \Gamma / k A_{j}\right)\right)$ account for mixed advection and diffusion. The argument to the exponential is the Péclet number $(P e)$, which is ratio of advective to diffusive flow. In the case that the advective flow is in the positive direction, the argument will be negative for the negative-facing boundary and positive for the positive-facing boundary. The factor can be interpreted as adjusting the length of diffusive transport according to the extent of drift current or bulk velocity. In the case that there is no bulk velocity, the factor is two; the length from the center of the region to the port is half of the length of the subregion along that axis. Under isothermal conditions, the equations reduce to Fick's law (in the case of chemical species) and Ohm's law (in the case of electrons or holes). In the case that $\mathrm{Pe} \rightarrow \infty$, the effort at the positive-facing boundary is equal to the value in the bulk of the region. That is, properties are propagated in the downstream direction. Meanwhile, the relationship between the effort of the negative-facing boundary is related to the effort in the bulk of the region by pure diffusion with the full length of the subregion. The relationships reverse when advective flow is in the opposite direction.

The exchange and transport equations for volume, momentum, and energy are similar to that for material.
If there is only diffusion, then the transport equations for transverse momentum, if included, reduce to the case of Couette flow. The transport equation for energy reduces to thermal conduction when there is no advection and the other efforts are uniform. It is differentially equivalent to Fourier's law. Otherwise, the case is thermal convection-combined advection and diffusion.

In the case of exchange rather than transport, the $A / L$ factor is combined as characteristic length $\left(L^{\star}\right)$. It must be calibrated by parameter identification or determined empirically.

The exchange equation for momentum, like the Stefan-Maxwell equation, describes the drag forces between species traveling at different velocities through a mixture [2, p. 538]. However, this approach more manageable. As stated by Cussler, the "StefanMaxwell equation is almost never used because it is difficult to solve mathematically, even in the simplest cases" [6].

The Onsager formulation allows gradient of one type of effort to affect the flow rate of quantities besides its conjugate pair. Advection is described in this manner; the same gradient that drives material flow 
also drives other flows. ${ }^{i}$ The difference in velocity normal to the face is coupled to the material flow rate in a reciprocal manner as the difference in chemical potential is coupled to the momentum flow rate.

The exchange/transport equations allow there to be storage within the region, even due to transport along a single axis, because the rates into two faces is not necessarily equal and opposite. In the case of pure diffusion, the rate of intake is proportionally to the second gradient of the effort. The rate balance or conservation equations are given by Equation 3. Einstein notation is used in the summations of the energy rate balance. The form of the energy equation follows from the Onsager approach [1].

\section{Discussion}

The exchange and transport parameters are cast in terms of resistivity instead of conductivity so that index reduction may be initiated by setting the resistance(s) to zero as final. A typical assumption is that all species (at least within a mixture) are at the same temperature. In addition, the total pressures of the species are expected to be the same after a very short time. If liquid water is added, it may be appropriate to assume that it is in equilibrium with the water vapor. With these assumptions, the number of degrees of freedom reduces to that given by Gibbs' phase rule. It states that the number of thermodynamic degrees of freedom is equal to two plus the number of species minus the number of phases [20], [1, pp. 24-49]. In the case of the assumptions that have been mentioned, the natural thermodynamic state variables would be temperature (the same for all species) and the particle numbers of each chemically independent species or group of species in phasic equilibrium. In the model, index reduction generally introduces nonlinear equations and there is a performance tradeoff between fewer states and fewer nonlinear equations.

\section{Conclusion}

The architecture and equations for the PEMFC model library have been described at a high level. The implementation is modular and flexible. The same approach would support other electrochemical devices such as batteries. The library is being refined and tested. Results will be given and discussed in a future publication

\footnotetext{
${ }^{\mathrm{i}}$ The factor which includes the exponential only amplifies or attenuates the effect of an effort gradient on its own conjugate.
}

after validation and calibration.

The library is being made available as open source and should appear on the Modelica website (www . modelica.org). Collaboration would be welcomed. 


\section{Nomenclature}

\section{Symbols}

\begin{tabular}{|c|c|}
\hline$A$ & Area $\left[\mathrm{L}^{2}\right]$ \\
\hline$c$ & Specific heat capacity [1] \\
\hline$U$ & Energy $\left[\mathrm{L}^{2} \mathrm{M} \mathrm{T}^{-2}\right]$ \\
\hline$k$ & Area factor $[1]$ \\
\hline$L$ & Length [L] \\
\hline$m$ & Specific mass [1] \\
\hline$m \Phi$ & Linear momentum $\left[\mathrm{L} \mathrm{M} \mathrm{T}^{-1}\right]$ \\
\hline$N$ & Particle number $[\mathrm{N}]$ \\
\hline$P$ & Pressure $\left[\mathrm{ML}^{-1} \mathrm{~T}^{-2}\right]$ \\
\hline$P e$ & Peclet number [1] \\
\hline$T$ & Temperature $\left[\mathrm{L}^{2} \mathrm{MN}^{-1} \mathrm{~T}^{-2}\right]$ \\
\hline$t$ & Time $[\mathrm{T}]$ \\
\hline$V$ & Volume $\left[\mathrm{L}^{3}\right]$ \\
\hline$v$ & Specific volume $\left[\mathrm{L}^{3} \mathrm{~N}^{-1}\right]$ \\
\hline $\mathbf{a}$ & Global acceleration $\left[\mathrm{L} \mathrm{T}^{-2}\right]$ \\
\hline$\beta$ & Onsager coupling coefficient [misc.] \\
\hline$\Gamma$ & Generalized resistivity $\left[\mathrm{L} \mathrm{T} \mathrm{N}^{-1}\right]$ \\
\hline$\mu$ & Chemical potential $\left[\mathrm{L}^{2} \mathrm{M} \mathrm{N}^{-1} \mathrm{~T}^{-2}\right]$ \\
\hline$\Phi$ & Particle number times velocity $\left[\mathrm{L} \mathrm{N} \mathrm{T}^{-1}\right]$ \\
\hline & Linear velocity $\left[\mathrm{L} \mathrm{T}^{-1}\right]$ \\
\hline
\end{tabular}

\section{Accents}

Flow rate of $\left[\times \mathrm{T}^{-1}\right]$

\section{Superscripts}

* Effective or characteristic

\section{Subscripts}

$\begin{array}{ll}i & - \text { of index } i \\ j & - \text { of index } j \\ N & - \text { of material } \\ P & - \text { at constant pressure } \\ V & - \text { of volume } \\ \Phi & \text { - of linear momentum } \\ U & \text { - of energy }\end{array}$

\section{Acknowledgments}

The authors wish to acknowledge support from the Presidential Fellowship of the Georgia Institute of Technology and the Robert G. Shackelford Fellowship of the Georgia Tech Research Institute.

\section{References}

[1] A. Bejan. Advanced Engineering Thermodynamics. John Wiley \& Sons, 3rd edition, 2006.

[2] R. B. Bird, W. E. Stewart, and E. N. Lightfoot. Transport Phenomena. John Wiley \& Sons, 2nd edition, 2002.

[3] W. Borutzky. Bond Graph Modelling of Engineering Systems: Theory, Applications and Software Support. Springer, 2011.

[4] K. A. Burke. Unitized regenerative fuel cell system development. NASA report TM-2003212739, Glenn Research Center, Cleveland, OH, Dec. 2003.

[5] F. E. Cellier and J. Greifeneder. Modeling chemical reactions in modelica by use of chemo-bonds. In F. Casella, editor, Proc. 7th Int. Modelica Conf., Como, Italy, Sep. 2009. Modelica Assoc., Linköping University Electronic Press.

[6] E. L. . Cussler. Diffusion: Mass Transfer in Fluid Systems. Cambridge University Press, 2nd edition, 1997.

[7] K. L. Davies and R. M. Moore. Object-oriented fuel cell model library. Electrochem. Soc. T., 11(1):797-808, Oct. 2007.

[8] K. L. Davies and R. M. Moore. PEMFCSim: A fuel cell model library in Modelica. In 31st Fuel Cell Seminar \& Exposition, San Antonio, TX, Oct. 2007.

[9] K. L. Davies, R. M. Moore, and G. Bender. Model library of polymer electrolyte membrane fuel cells for system hardware and control design. In F. Casella, editor, Proc. 7th Int. Modelica Conf., Como, Italy, Sep. 2009. Modelica Assoc., Linköping University Electronic Press.

[10] K. L. Davies and C. J. Paredis. Natural unit representation in Modelica. In Proc. 9th Int. Modelica Conf., Munich, Germany, Sep. 2012 (submitted). Modelica Assoc.

[11] J. H. Dymond, K. N. Marsh, R. C. Wilhoit, and K. C. Wong. Virial Coefficients of Pure Gases. Numerical Data and Functional Relationships in Science and Technology. Springer-Verlag, 2002.

[12] Dynasim AB. Dymola: Dynamic Modeling Laboratory, Mar. 2010. Ver. 7.4. 
[13] F. P. Incropera and D. P. DeWitt. Fundamentals of Heat and Mass Transfer. John Wiley \& Sons, 5th edition, 2002.

[14] J. Larminie and A. Dicks. Fuel Cell Systems Explained. John Wiley \& Sons, 2nd edition, 2003.

[15] C. Mattiussi. The finite volume, finite element, and finite difference methods as numerical methods for physical field problems. volume 113 of Advances in Imaging and Electron Physics, pages 1-146. Elsevier Academic Press, 2000.

[16] B. J. McBride, M. J. Zehe, and S. Gordon. NASA Glenn coefficients for calculating thermodynamic properties of individual species. NASA report TP-2002-211556, Glenn Research Center, Cleveland, OH, Sep. 2002.

[17] B. A. McCain, A. G. Stefanopoulou, and K. R. Butts. A study toward minimum spatial discretization of a fuel cell dynamics model. In Proc. Int. Mech. Eng. Congr. Exposition (IMECE2006), number IMECE2006-14509, Chicago, IL, Nov. 2006. ASME.

[18] D. A. McKay, W. T. Ott, and A. G. Stefanopoulou. Modeling, parameter identification, and validation of water dynamics for a fuel cell stack. In Conf. on Fuel Cell Science, Engineering and Technology, Orlando, FL, Nov. 2005. ASME. FUELCELL2005-81484.

[19] Modelica Association. Modelica Standard Library. http: //www.modelica.org/ libraries/Modelica, Dec. $2009 . \quad$ Ver. 3.1 .

[20] M. J. Moran and H. N. Shapiro. Fundamentals of Engineering Thermodynamics. John Wiley \& Sons, 6th edition, 2008.

[21] R. D. Present. Kinetic Theory of Gases. McGraw-Hill, 1958.

[22] M. A. Rubio, A. Urquia, L. Gonzájlez, D. Guinea, and S. Dormido. FuelCellLib: A modelica library for modeling of fuel cells. In Proc. 4th Int. Modelica Conf., HamburgHarburg, Germany, Mar. 2005. Modelica Association.

[23] M. A. Rubio, A. Urquiaa, and S. Dormidoa. Dynamic modelling of PEM fuel cells using the FuelCellLib Modelica library. Math. Comp. Model. Dyn., 16(3):165-194, Jun. 2010.
[24] A. Salogni and P. Colonna. Modeling of solid oxide fuel cells for dynamic simulations of integrated systems. Appl. Therm. Eng., 30(5):464477, 2010.

[25] R. A. Svehla. Transport coefficients for the nasa lewis chemical equilibrium program. NASA Technical Memorandum NASA, Lewis Research Center, Cleveland, OH, Apr. 1995.

[26] U.S. Department of Energy. Hydrogen, fuel cells \& infrastructure technologies program: Multiyear research, development and demonstration plan. Technical report, Energy Efficiency and Renewable Energy, Oct. 2007. Section 3.4: Fuel Cells.

[27] N. Wagner, W. Schnurnberger, B. Mueller, and M. Lang. Electrochemical impedance spectra of solid-oxide fuel cells and polymer membrane fuel cells. Electrochim. Acta, 43(24):3785-3793, 1998.

[28] A. Z. Weber, R. M. Darling, and J. S. Newman. Modeling two-phase behavior in PEFCs. J. Electrochem. Soc., 151(10):A1715-A1727, 2004.

[29] K. W. Woo and S. I. Yeo. Dalton's Law vs. Amagat's Law for the mixture of real gases. SNU J. Educ. Res., 5:127-134, 1995. 
